Disponível em

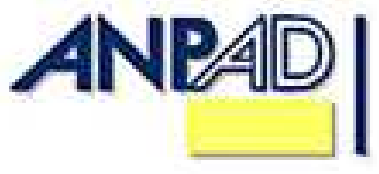

http://www.anpad.org.br/rac

RAC, Rio de Janeiro, v. 17, n. 2, art. 5, pp. 218-238, Mar./Abr. 2013

(cc) EY-NG

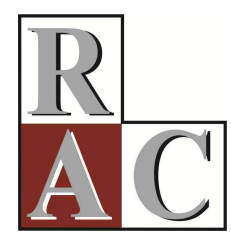

\title{
Sobrequalificação no Trabalho e sua Influência sobre Atitudes e Comportamentos
}

\author{
Overqualification at Work and Its Influence on Attitudes and Behaviors
}

Cristiano Oliveira Maciel

E-mail: cristiano.maciel@pucpr.br Pontifícia Universidade Católica do Paraná - PUCPR Rua Espírito Santo, 411, 80630-200, Curitiba, PR, Brasil.

Camila Camargo E-mail: caca.adm@gmail.com Universidade Federal do Paraná - UFPR Rua Espírito Santo, 411, 80630-200, Curitiba, PR, Brasil. 


\title{
Resumo
}

O interesse pela noção de competência tem contribuído para que o conceito de sobrequalificação no trabalho permaneça à margem da corrente principal no campo dos estudos organizacionais. Para explorar essa lacuna, delineou-se o seguinte objetivo de pesquisa: verificar a influência da sobrequalificação percebida no trabalho sobre satisfação, intenção de saída e cooperação do indivíduo na organização. Após desenvolvimento das hipóteses, foram investigados, por meio de um survey, 331 trabalhadores de oito organizações. As hipóteses foram testadas por meio de um Modelo de Equações Estruturais. Os resultados apontam que, das duas dimensões da Sobrequalificação, Incongruência (habilidades além do necessário para a função) e Contracrescimento (restrições para desenvolvimento e uso das habilidades), é o fator Contracrescimento que influencia mais significativamente a Satisfação, a Intenção de Saída e o Comportamento Cooperativo. Contrariamente a uma das hipóteses, verificou-se que a dimensão Incongruência influencia positivamente na cooperação do indivíduo com a organização.

Palavras-chave: sobrequalificação; satisfação; intenção de saída; cooperação; setor de serviços.

\begin{abstract}
In the organizational studies field, interest in the notion of competence has contributed to keeping the concept of overqualification at work outside of the mainstream. The research objective of this investigation intends to fill this gap: to verify the influence an individual's Perceived Overqualification at work has on Satisfaction, Intention to leave an organization, and Cooperation within an organization. After developing hypotheses, 331 workers from 8 organizations were investigated via survey and the hypotheses were tested using Structural Equation Model. The results point out that, between the two Overqualification dimensions - Mismatch (having more skills than necessary for a function) and No Growth (restrictions to the development and use of skills), it is the No Growth factor that more significantly influences Satisfaction, Intention to leave an organization and Cooperative behavior. We further verified that the Mismatch dimension positively influences an individual's cooperation within an organization.
\end{abstract}

Key words: overqualification; satisfaction; intention to leave an organization; cooperation; service sector. 


\section{Introdução}

A avaliação que os indivíduos fazem acerca de quanto estão sendo subutilizados na execução de suas atividades de trabalho é frequentemente identificada, na literatura, como sobrequalificação percebida, subemprego ou superqualificação (McKee-Ryan \& Harvey, 2011; Scurry \& Blenkinsopp, 2011). A percepção de sobrequalificação no trabalho é fenômeno que emerge normalmente de duas situações. A primeira caracteriza-se quando o indivíduo percebe incongruências a partir de altos graus de sofisticação de seus conhecimentos e habilidades contrastados com exigências mínimas dessas habilidades no desempenho de suas funções. A segunda, quando a organização restringe o uso de suas qualificações e impossibilita o desenvolvimento de novas habilidades (W. R. Johnson, Morrow, \& Johnson, 2002).

A importância desse tema cresce na medida em que não se constata um movimento de enriquecimento do trabalho (atividades que ofereçam maior autonomia) na mesma intensidade do desenvolvimento de conhecimentos e habilidades dos indivíduos na maioria das sociedades, sobretudo, em casos de significativos aumentos na oferta de ensino superior (Fine, 2007; Fine \& Nevo, 2008; G. J. Johnson \& Johnson, 1999, 2000), como vem ocorrendo no Brasil ou, mais acentuadamente, nos países asiáticos. McKee-Ryan e Harvey (2011), por exemplo, apontam estatísticas de que até $30 \%$ da força de trabalho ativa norte-americana sustentam essa percepção sobre si em relação ao seu trabalho. Entre os recém-graduados no ensino superior, na Europa, os dados apontam que metade dessa população se percebe enquadrada nessa mesma situação (McKee-Ryan \& Harvey, 2011; Scurry \& Blenkinsopp, 2011). Em momentos de escassez de empregos, como afirmam McKee-Ryan e Harvey (2011) e Walling e Clancy (2010), a percepção de sobrequalificação acentuase na medida em que as alternativas de trabalho são mais reduzidas e são aceitos trabalhos menos qualificados.

Nesse sentido, é surpreendente que os periódicos da área de gestão com maior fator de impacto, como Academy of Management Journal e Academy of Management Review, apresentem apenas dois trabalhos pertinentes ao tema; ou mesmo que revistas mais especializadas, como Journal of Organizational Behavior, não apresentem sequer uma dezena de artigos que estejam voltados para o tema da sobrequalificação (overqualification/underemployment).

Apesar de parecer fundamental a influência desse tipo de julgamento sobre atitudes e comportamentos do indivíduo com a organização, é a sociologia e, sobretudo, a economia que se têm debruçado mais detidamente sobre esse fenômeno (Anda \& Sobczak, 2011; Walling \& Clancy, 2010). Mas a preocupação de sociólogos e economistas com esse fenômeno está mais restrita às suas consequências sobre o mercado de trabalho de forma agregada e não sobre o comportamento dos indivíduos no contexto organizacional.

Tal assertiva merece destaque ao considerar o número de fatores atitudinais e comportamentais possivelmente influenciados por esse constructo (Brynin \& Longhi, 2009; Budría, 2011; Erdogan \& Bauer, 2009; Wilkins, 2009). G. J. Johnson e Johnson (2000), por exemplo, posicionam a variação no grau de satisfação com o trabalho como imediato e principal consequente da sobrequalificação percebida. Por extensão, é razoável supor que a intenção de saída seja influenciada também pela sobrequalificação, tendo a satisfação como uma variável mediadora. Mas vale advertir que a relação exposta por G. J. Johnson e Johnson (2000) contempla apenas o plano de atitude e, para que a pesquisa atinente a esse tópico possa avançar, é preciso investigar a influência da sobrequalificação também sobre aspectos comportamentais, mais especificamente, sobre a ação dos indivíduos no trabalho.

Assim, ao considerar os argumentos expostos anteriormente e com intenção de fazer avançar no sentido de desenvolver uma teoria da sobrequalificação no trabalho (G. J. Johnson \& Johnson, 2000), foi proposto aqui o seguinte objetivo de pesquisa: verificar a influência da sobrequalificação percebida no trabalho sobre satisfação, intenção de saída e cooperação do indivíduo na organização. Sobrequalificação, Satisfação e Intenção de Saída são as variáveis de atitudes; e a Cooperação, a variável comportamental. Assim, a principal contribuição do artigo centra na exploração do tema 
sobrequalificação ao associá-lo a constructos atitudinais e também a um fator comportamental simultaneamente num mesmo modelo de análise.

A pesquisa ocorreu por meio de um levantamento (survey) com 331 trabalhadores de oito organizações do setor de serviços de Curitiba (PR). O estudo é apresentado nos seguintes tópicos, após esta introdução: quadro teórico-empírico e hipóteses; procedimentos metodológicos; análise dos dados; discussão dos resultados; e, considerações finais.

\section{Quadro Teórico-Empírico de Referência e Hipóteses}

O referencial teórico-empírico no qual se apoia esta investigação está dividido em dois subitens: sobrequalificação percebida e consequentes atitudinais e comportamentais da sobrequalificação.

\section{Sobrequalificação percebida}

Diferentemente de sociólogos e economistas, a noção de sobrequalificação no trabalho, tendo as organizações como contexto imediato, tem sido mais intensamente explorada no campo da psicologia, principalmente por dois professores da Iowa University, W. Roy Johnson e Gloria Jones Johnson (e.g., W. R. Johnson et al., 2002; G. J. Johnson \& Johnson 1999, 2000). Já na área de comportamento organizacional e gestão de pessoas, o interesse dos pesquisadores sobre o potencial de desempenho do indivíduo no trabalho parece ter privilegiado o conceito de competência.

Entretanto, tendo em vista a complexidade de mensuração das competências (entendidas como a configuração de conhecimentos e habilidades) (Sandberg, 2000), ressalta-se que o constructo sobrequalificação percebida apresenta algumas vantagens, como, por exemplo, mais fácil operacionalização em comparação às definições de competências individuais e organizacionais (Frei \& Sousa-Poza, 2012; Sugiyarto, 2008). Ademais, as definições de competência e sobrequalificação percebida não são incorporadas uma pela outra, são conceitos apenas relacionados, mas diferentes um do outro. Como apontou Sandberg (2000, p. 9), após substancial revisão da literatura de base racionalista e interpretativa, o conceito de "competência humana no trabalho" é definido comumente como aqueles conhecimentos e habilidades que as pessoas usam quando estão trabalhando. No mesmo sentido, os trabalhos de Mansfield (1996) e Noor e Dola (2009) servem como outros exemplos de definição do conceito de competência a partir da configuração de conhecimentos e habilidades. No Brasil, o trabalho de Castro e Borges-Andrade (2004) também enfatiza a junção de conhecimentos e habilidades, particularmente na operacionalização conjunta dessas dimensões, mas vai além ao compor o conceito com algumas características atitudinais também. Essa definição revela a constituição situacional e específica de conhecimentos e habilidades para cada tipo de trabalho no tempo e no espaço. Tal definição indica maior complexidade da noção de competência ao exigir o delineamento situacional (em cada caso) de habilidades e conhecimentos. Já a noção de sobrequalificação percebida é, em termos operacionais, mais simples, pois permite restringir-se ao julgamento que o próprio trabalhador faz da distância entre as suas capacidades e as exigências de suas atividades. Nesse sentido, não importa se o trabalhador compara as exigências de seu trabalho com o que ele chama de competência, capacidades ou habilidades, mas sim se seu julgamento é de que ele tem qualificações superiores ao utilizado ou não. Apesar de existirem propostas semelhantes de pesquisa sobre a falta de utilização do potencial do indivíduo no trabalho, essas figuram-se sob a égide de conceitos como subutilização de habilidades, subemprego e falta de oportunidades para crescimento e mudança (Livingstone, 2010; McKee-Ryan \& Harvey, 2011; Scurry \& Blenkinsopp, 2011). Mas esses termos são definidos e operacionalizados de modos imprecisos, com sobreposições em diversos casos e grande variação na utilização de um mesmo conceito em outros (W. R. Johnson et al., 2002).

Decorre daí a proposta de W. R. Johnson, Morrow e Johnson (2002), como destacado anteriormente, para definição da sobrequalificação no trabalho a partir de duas dimensões: julgamento do indivíduo de que ele possui qualificações acima do necessário para o trabalho que executa 
(Mismatch) (Weststar, 2009) e percepção de que enfrenta oportunidades limitadas para adquirir e usar novas habilidades no desempenho de suas funções (No-Growth). Essas duas dimensões (Mismatch e No-Growth) foram aqui livremente traduzidas, respectivamente, pelos seguintes termos: Incongruência Percebida e Contracrescimento Percebido.

A dimensão Incongruência Percebida (Mismatch) tem origem no exercício de comparação de vários elementos. Esse processo varia de indivíduo para indivíduo no que se refere à avaliação que o mesmo faz de suas próprias habilidades, seu grau de instrução e suas experiências profissionais contrapostas aos desafios e grau de exigência das atividades desempenhadas em sua função. Nessa avaliação o ponto de partida é o conjunto de qualificações do indivíduo (G. J. Johnson \& Johnson, 1999, 2000).

Na dimensão Contracrescimento Percebido (No-Growth), o ponto de partida da avaliação é a dinâmica oferecida ou restringida pela própria organização. O que é considerado por aquele que desempenha uma dada atividade é o quanto a organização possibilita desafios e inovações que conduzam ao aprimoramento das habilidades já controladas, bem como conquistas de outras novas (G. J. Johnson \& Johnson, 1999, 2000).

\section{Consequentes atitudinais e comportamentais da sobrequalificação}

Extrai-se da discussão anterior que, por um lado, a sobrequalificação percebida tem origem principalmente em fatores pessoais, como a própria evolução profissional, educacional e de experiências passadas do indivíduo (Green \& Zhu, 2010; Watt \& Hargis, 2010) ou até mesmo em características demográficas (e.g., idade e estado civil). Por outro lado, a sobrequalificação também ocorre em função de aspectos organizacionais, que impactam no desenvolvimento de novas habilidades. McKee-Ryan e Harvey (2011), por exemplo, destacam um estudo em que expatriados de maior nível hierárquico apresentaram menor percepção de sobrequalificação. Esses autores ressaltam que a rotinização da atividade altera a natureza do trabalho. Assim, se o trabalho é mais desafiador, ele exige mais das habilidades do indivíduo e isso atenua a sobrequalificação.

No mesmo sentido, Erdogan e Bauer (2009) afirmam que as organizações dispõem de alternativas para mudanças no contexto organizacional e, mais especificamente, no próprio trabalho que podem moderar a relação entre a sobrequalificação e fatores como satisfação do indivíduo e seu desempenho. Esses autores identificaram que o empowerment (enquanto um mecanismo de delegação de poder) opera como base para atribuição de maior desafio das atividades e crescimento das responsabilidades no trabalho, exercendo influência indireta sobre a satisfação do indivíduo com o trabalho e sua intenção de saída da organização.

Mas, para além dos fatores responsáveis pela percepção de sobrequalificação, interessa, principalmente às organizações, o impacto que esse fenômeno exerce em aspectos atitudinais (opiniões, avaliações e intenções) e comportamentais (ações) (Erdogan \& Bauer, 2009; Fine \& Nevo, 2008; Green \& Zhu, 2010; G. J. Johnson \& Johnson, 2002).

O primeiro aspecto atitudinal que figura como consequente direto da sobrequalificação no trabalho é a satisfação. Essa relação foi inicialmente explorada por G. J. Johnson e Johnson (2000). Entretanto esses autores investigaram a relação entre sobrequalificação e algumas das dimensões do constructo satisfação ao invés do constructo satisfação global com o trabalho, o qual contempla necessariamente fatores cognitivos e emocionais.

Contrariamente a G. J. Johnson e Johnson (2000), advoga-se aqui que a mensuração da satisfação do indivíduo em relação a aspectos específicos do trabalho reflete a qualidade percebida (desempenho) de tais atributos e não a resposta atitudinal global (soma dos julgamentos de ordem instrumental e também afetiva) no que concerne ao trabalho como um todo. Whitman, Roy e Viswesvaran (2010), assim como Schwepker (2001) e Rusbult e Farrel (1983), apresentam a satisfação no trabalho como a soma de componentes de resposta cognitiva e componentes de resposta afetiva que são formados durante e após a experiência de execução de atividades produtivas. Vale destacar, como 
apresenta Spector (2005), que essa definição não enfatiza os aspectos disposicionais do indivíduo (i.e., sua personalidade e a influência dela na satisfação), pois alguns seres humanos apresentam tendências a julgar mais satisfatoriamente o trabalho do que outros.

Assim, a satisfação, enquanto resposta cognitiva e afetiva, não pode ser medida diretamente em relação aos atributos organizacionais, pois o indivíduo, em seu processo de avaliação do trabalho como um todo, pode ser influenciado por dissonância cognitiva. Por conta de outros motivos que o impelem a ficar na organização, ele não admite sua insatisfação, superestima seu nível de satisfação para se convencer de que o trabalho supre suas necessidades e, assim, mascara o conflito entre sua vontade ou necessidade de permanência e a insatisfação com o trabalho (Laschinger, Finegan, Shamian, \& Wilk, 2004). Logo, seria mais adequado considerar a satisfação como uma avaliação global (Ferrat, 1981). Alguns outros estudos também identificaram a influência da sobrequalificação sobre a insatisfação com o trabalho (e.g., Green \& Zhu, 2010).

Assim, é possível argumentar que a satisfação do indivíduo, enquanto uma resposta global de natureza afetiva e cognitiva (instrumental) em relação ao trabalho, figure como um elemento a ser influenciado pelas duas dimensões da sobrequalificação no trabalho (Incongruência e Contracrescimento). E decorre daí as primeiras hipóteses do presente estudo:

$\mathbf{H}_{1 \mathbf{a}}$ : A Incongruência percebida entre habilidades pessoais e demandas das atividades do indivíduo no trabalho influencia negativamente sua satisfação com o trabalho.

$\mathbf{H}_{1 \mathbf{b}}$ : O Contracrescimento, que se refere às limitações organizacionais que impedem o desenvolvimento das habilidades do indivíduo no trabalho, influencia negativamente sua satisfação com o trabalho.

Outro constructo que pode ser influenciado pela sobrequalificação no trabalho é a intenção de saída da organização, porque este é fortemente associado à satisfação. Mais especificamente, Steel (2002) apresenta a intenção de saída como o produto de um processo decisório essencialmente linear que reflete a vontade do indivíduo em não pertencer mais à sua organização. Bowen (1982), em texto clássico sobre a intenção de saída, emprega a definição de intenção de saída como o desejo percebido de movimento para fora da organização. Bowen (1982), também, apontou vários estudos que comprovaram a relação significativa entre a intenção de saída e a saída efetiva da organização, destacando, assim, a importância em considerar esse constructo em relação ao turnover e a outros fatores atitudinais e comportamentais do indivíduo no trabalho. Essa vontade de desligamento físico e legal da organização tem seus imperativos em diversos fatores, tanto sociais, econômicos e culturais quanto organizacionais (e.g., tipo de atividade desempenhada no trabalho).

Em termos organizacionais, pode-se esperar que a incompatibilidade (Incongruência) entre habilidades do indivíduo e as exigências de suas funções e a falta de oportunidades de desenvolvimento de novas qualificações (Contracrescimento) operem como importantes antecedentes da sua intenção de saída. Essas duas situações são apresentadas pelas seguintes hipóteses:

$\mathbf{H}_{2 \mathbf{a}}$ : A Incongruência percebida entre habilidades pessoais e demandas das atividades do indivíduo no trabalho influencia positivamente sua intenção de saída da organização.

$\mathbf{H}_{2 \mathrm{~b}}$ : O Contracrescimento, que se refere às limitações organizacionais que impedem o desenvolvimento das habilidades do indivíduo no trabalho, influencia positivamente sua intenção de saída da organização.

Reconhecendo a relação que se estabelece entre satisfação do indivíduo com o trabalho e sua intenção de saída da organização, espera-se que esses elementos apresentem forte associação, mas com sentido negativo. Diferentemente das demais hipóteses do presente estudo, essa relação é bemdocumentada na literatura e reiteradamente comprovada em estudos empíricos (Cotton \& Tuttle, 1986; Mobley, 1982). De tal assertiva foi desenvolvida a próxima hipótese: 
$\mathbf{H}_{3}$ : A satisfação do indivíduo com o trabalho influencia negativamente sua intenção de saída da organização.

Como mencionado, as hipóteses apresentadas até aqui versam sobre elementos de natureza atitudinal e não comportamental. Entretanto variáveis de ordem comportamental (e.g., produtividade ou inovação) exigem observar métricas relacionadas a ações específicas do indivíduo no trabalho, o que traz implicações metodológicas que impedem a realização da maioria dos estudos que fazem uso de dados coletados a partir de questionários.

Desse modo, é conveniente lançar mão de levantamentos atinentes a padrões de ação ou comportamentais no trabalho, como é o caso da cooperação. A noção de cooperação é frequentemente explorada via teorias da economia (e.g., Axelrod \& Dion, 1988; Phua, 2004). Entretanto a ideia de ação conjunta na economia é tratada estritamente a partir de pressupostos do agente econômico neoclássico e outros fatores atitudinais são desconsiderados. Já na área de organizações, a cooperação é definida como esforços distintos, similares e complementares para consecução de um objetivo coletivo e aparece relacionada a outros elementos de ordem atitudinal, como, por exemplo, cultura, personalidade, identidade e identificação (Chatman \& Barsade, 1995; Dukerich, Golden, \& Shortell, 2002).

Nesse sentido, a ideia de comportamento cooperativo nos sistemas organizacionais, conforme Barnard (1979), é interessante por possibilitar a mensuração de um padrão comportamental comparado aos demais membros organizacionais a partir do julgamento do próprio avaliador (e.g., funcionário). Assim, questões de natureza atitudinal atuam como antecedentes de padrões comportamentais de cooperação com a organização e, de modo mais restrito, com colegas de grupo de atuação. Logo, o comportamento cooperativo do indivíduo com a organização, definido como a ação conjunta e na mesma direção de outros membros organizacionais para alcance de determinado propósito (Barnard, 1979; Chen, Xie, \& Chang, 2011; Jones \& George, 1998), também, é considerado mais especificamente como um consequente da sobrequalificação, assim como de outras motivações individuais no trabalho (e.g., satisfação). Na medida em que se entendem as dimensões Incongruência e Contracrescimento como fatores que operam no sentido contrário ao atendimento das necessidades humanas nas organizações, o constructo sobrequalificação figura então como fonte de satisfação e insatisfação. Ressalta Barnard (1979, p, 80): "Se um indivíduo acha que seus motivos estão sendo satisfeitos pelo que ele faz, ele continua o seu esforço cooperativo; em caso contrário, ele não o faz". Desses argumentos extraem-se as hipóteses $\mathrm{H}_{4 a}, \mathrm{H}_{4 \mathrm{~b}}$ e $\mathrm{H}_{5}$ :

$\mathbf{H}_{4 \mathbf{a}}$ : A Incongruência percebida entre habilidades pessoais e demandas das atividades do indivíduo no trabalho influencia negativamente seu comportamento cooperativo.

$\mathbf{H}_{\mathbf{4}}$ : $\mathrm{O}$ Contracrescimento, que se refere às limitações organizacionais que impedem o desenvolvimento das habilidades do indivíduo no trabalho, influencia negativamente seu comportamento cooperativo.

$\mathbf{H}_{5}$ : A satisfação do indivíduo com o trabalho influencia positivamente seu comportamento cooperativo.

Tradicionalmente, o principal elemento que figura como resultado da intenção de saída do indivíduo no trabalho é sua ação, sobretudo em termos de produtividade e outros tipos de comportamento em âmbito individual, com reflexos no desempenho organizacional (Mobley, 1982). Como já foi exposto, a cooperação é tida, no presente artigo, como importante variável comportamental que pode ser influenciada também pela intenção de saída da organização. Da exposição dessa relação, apresenta-se a hipótese $\mathrm{H}_{6}$ :

$\mathbf{H}_{6}$ : A intenção de saída da organização influencia negativamente o comportamento cooperativo do indivíduo no trabalho. 


\section{Procedimentos Metodológicos}

$\mathrm{Na}$ descrição dos procedimentos metodológicos seguidos para consecução do objetivo do presente trabalho, constam os seguintes tópicos: delineamento e delimitação do estudo, escalas de mensuração e tratamento dos dados.

\section{Delineamento e delimitação}

Em relação ao delineamento, optou-se pela realização de um levantamento (survey) para a coleta de dados (Kerlinger, 2003). O estudo adota corte seccional em sua dimensão temporal, e a unidade de análise é o indivíduo (Babbie, 1998).

No que concerne à delimitação do estudo, o universo de pesquisa foi composto por 991 funcionários empregados em oito organizações do setor de serviços de Curitiba (PR). Essas organizações são dos setores de: call center, escola de condutores, lojas de varejo de serviços e organizações de serviços de informática. Dada à impossibilidade de realização de um censo, sobretudo em função da participação voluntária para preenchimento do questionário, tempo e custos, fez-se uso de uma amostragem não probabilística por conveniência. Os questionários foram impressos e distribuídos para os funcionários ao longo de dois meses. Quando da distribuição dos questionários, foi comunicado aos participantes que eles deveriam fazer o depósito do questionário em uma caixa que só poderia ser aberta pelos pesquisadores e, com isso, não seria realizada qualquer forma de identificação durante ou após a pesquisa encerrada. Terminada a coleta dos dados, o total de 351 questionários foi analisado em termos de valores perdidos (missing values) e falta de variação mínima nas respostas. Depois da exclusão de alguns questionários, a amostra final foi composta por 331 questionários válidos.

\section{Escalas de mensuração}

Para caracterização da amostra foram empregadas as variáveis; idade, sexo, cargo, estado civil e tempo na empresa. Para mensuração da sobrequalificação percebida, foi utilizada uma adaptação da Perceived Overqualification Scale de W. R. Johnson et al. (2002), que é composta por duas dimensões: Perceived No-Growth (Contracrescimento) e Perceived Mismatch (Incongruência).

A escala utilizada por esses autores foi composta por um conjunto de cinco itens em cada dimensão. Entretanto, com o objetivo de estruturar um questionário menos extenso, foram escolhidos apenas três itens de cada fator. Os indicadores escolhidos foram aqueles com maior carregamento nas Análises Fatoriais Confirmatórias e que se mostravam redundantes. Os itens foram traduzidos para o português e, posteriormente, um estudante de mestrado que passou alguns anos nos EUA verteu a tradução ao inglês para que esse resultado fosse comparado com os itens da escala original para assegurar a validade da tradução.

As escalas de satisfação, intenção de saída e cooperação foram desenvolvidas a partir das definições constitutivas dos constructos encontradas na literatura. Tratou-se a estrutura fatorial de cada uma dessas medidas como unidimensional. Com vistas a manter a parcimônia no número de itens de cada constructo, foram utilizados apenas três indicadores para cada um dos fatores. A satisfação foi avaliada de forma global e não por meio da satisfação em relação a alguns dos aspectos do trabalho. Como destaca Ferrat (1981), essas duas formas não são equivalentes. A escala de cooperação foi desenvolvida a partir do conceito de Barnard (1979) de modo a descrever a posteriori um padrão comportamental. O desenvolvimento de novos indicadores para esses constructos possibilitou dispensar o processo de tradução reversa das escalas. Todos os itens foram avaliados por meio de escala de Likert de 5 pontos (1 - discordo totalmente a 5 - concordo totalmente). As descrições dos indicadores de cada constructo constam no Apêndice. 


\section{Tratamento dos dados}

A fase de tratamento de dados teve início pela análise de outliers e de valores extremos. Na sequência, foram verificadas normalidade, curtose e assimetria das variáveis de modo a garantir a não violação dos pressupostos da análise estatística multivariada.

Para validação das escalas utilizadas no presente estudo, optou-se por iniciá-la com a verificação da estabilidade dos indicadores em seus respectivos fatores por meio da Análise Fatorial Exploratória (AFE), dividindo a base de dados em duas subamostras: amostra de teste $(n=168)$ e amostra de validação (163). A lógica desse exercício é verificar se, em diferentes amostras (amostra de teste e de validação), os indicadores de cada constructo permanecem em seus respectivos fatores derivados da Análise Fatorial Exploratória (AFE) e com consistência interna (Alfa de Cronbach) satisfatória (acima de 0,70) (Hair, Anderson, Tatham, \& Black, 1995; Kolenikov, Steinley, \& Thombs, 2010) nos dois conjuntos de observações.

Verificada a estabilidade dos indicadores em seus fatores previamente definidos e sua consistência interna nas amostras de teste e de validação o mesmo procedimento foi realizado para a base de dados completa. O passo seguinte consistiu na aplicação de uma Análise Fatorial Confirmatória (AFC), via Modelagem de Equações Estruturais, para as 331 observações.

$\mathrm{Na}$ sequência, foram calculados os índices de Confiabilidade Composta e Variância Média Extraída (AVE), para, então, ser avaliada a validade convergente e discriminante das medidas. E, por meio do modelo estrutural completo, foram testadas as hipóteses e avaliadas suas estimativas. Um resumo dos procedimentos seguidos no tratamento dos dados com apoio dos programas SPSS ${ }^{\circledR}$ (Statistical Package for the Social Sciences) 16 e AMOS ${ }^{\mathrm{TM}} 16$ consta na Figura 1.

\begin{tabular}{|c|c|}
\hline $\begin{array}{l}\text { Análise de outliers e valores extremos da } \\
\text { base de dados }(n=331) \text {. }\end{array}$ & $\begin{array}{l}\text { Análise Fatorial Exploratória na base de } \\
\text { dados completa }(n=331) \text {. }\end{array}$ \\
\hline 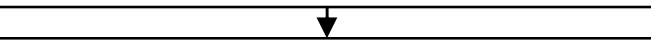 & 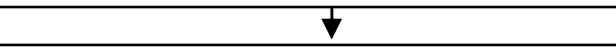 \\
\hline $\begin{array}{l}\text { Análise de normalidade, curtose e assimetria } \\
(\mathrm{n}=331) \text {. }\end{array}$ & Análise Fatorial Confirmatória $(\mathrm{n}=331)$. \\
\hline 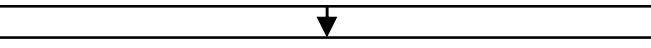 & $\frac{1}{x}$ \\
\hline $\begin{array}{l}\text { Divisão da base de dados em amostra de teste } \\
(n=168) \text { e de validação }(n=163) .\end{array}$ & $\begin{array}{l}\text { Cálculo de confiabilidade, validade } \\
\text { convergente e discriminante }(n=331) \text {. }\end{array}$ \\
\hline$\frac{1}{1}$ & $\frac{1}{7}$ \\
\hline $\begin{array}{l}\text { Análise Fatorial para verificação da } \\
\text { estabilidade dos fatores }(n=168) \text { e }(n=163) \text {. }\end{array}$ & $\begin{array}{l}\text { Avaliação do Modelo Estrutural Proposto e } \\
\text { do Modelo Rival }(n=331) \text {. }\end{array}$ \\
\hline 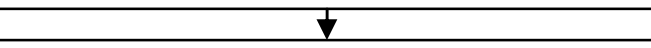 & 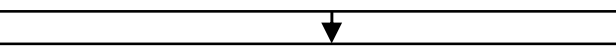 \\
\hline $\begin{array}{l}\text { Teste de consistência interna dos fatores - } \\
\text { amostras teste }(n=168) \text { e validação }(n=163) \text {. }\end{array}$ & $\begin{array}{l}\text { Teste das hipóteses: análise das estimativas } \\
\text { do Modelo Estrutural }(\mathrm{n}=331) \text {. }\end{array}$ \\
\hline
\end{tabular}

Figura 1. Procedimentos na Etapa de Tratamento dos Dados. Fonte: elaborada pelos autores.

Os resultados dos procedimentos seguidos na análise dos dados são apresentados no próximo tópico do artigo. 


\section{Análise dos Dados}

A amostra final da pesquisa $(n=331)$ apresentou as seguintes características: $51,4 \%$ mulheres e $48,6 \%$ homens, $36,6 \%$ são casados e $63,4 \%$ solteiros, $63,8 \%$ encontram-se em cargo operacional, $18,9 \%$ ocupam posto de supervisão ou coordenação e 17,3\% estão em cargos superiores. A média de idade dos respondentes é de 27,83 anos (desvio padrão $=8,66$, curtose $=2,6$ e assimetria $=1,24$ ), e a média de tempo na empresa é 4,10 anos (desvio padrão $=5,41$, curtose $=10,48$ e assimetria $=2,86$ ).

$\mathrm{Na}$ análise exploratória para verificação da qualidade dos dados, verificou-se que nenhuma das variáveis intervalares apresentou mais que oito valores perdidos (missing values), o que representa ausência não superior a $0,02 \%$ para cada indicador do questionário. Essa taxa de valores perdidos permitiu a imputação da média nos casos omissos. Na busca de valores atípicos, foram detectados dez outliers e nenhum valor extremo. Na comparação das análises, com e sem exclusão desses outliers, não foi identificada diferença nos resultados, logo, optou-se por manter as observações na base de dados para as análises seguintes.

Para teste da normalidade das variáveis, foi aplicado o teste Kolmogorov-Smirnov a um p-value $<0,05$, em que todas as variáveis intervalares se mostraram não normais, contudo a inspeção visual dos histogramas atestou que a distribuição dos dados não apresentava desvios significativos. Na sequência, foram examinados ainda os índices de curtose (excesso de curtose, pois o SPSS centra o valor de curtose, ou seja, assume zero (0) como curtose da curva normal) e assimetria. A curtose variou de $-1,351$ a 0,906 e a assimetria esteve entre $-0,966$ e 0,217. Esses valores possibilitam a aplicação de técnicas multivariadas para tratamento dos dados. Schumacker e Lomax (2004) afirmam que curtose e assimetria entre $\pm 1,5$ não impedem a aplicação de técnicas estatísticas que pressuponham normalidade.

Para aplicação do procedimento de análise da estabilidade dos indicadores em seus respectivos fatores, a base de dados $(\mathrm{n}=331)$ foi dividida em duas amostras; amostra de teste e de validação. A amostra de teste foi composta por 168 observações; e a de validação, por 163 respondentes. Esse procedimento possibilitou a aplicação da Análise Fatorial Exploratória (AFE) em cada subamostra para exame das dimensões e do coeficiente de consistência interna (Alfa de Cronbach).

A aplicação da Análise Fatorial Exploratória confirmou a estabilidade de todos os indicadores em seus respectivos fatores, conforme é previsto para cada escala, nas duas amostras (teste e validação). Na amostra teste ( $n=168)$, o Alfa de Cronbach variou entre 0,784 e 0,935 e na amostra de validação $(\mathrm{n}=163)$ de 0,777 a 0,956 . Comprovada a estabilidade dos indicadores em diferentes subamostras o mesmo processo foi aplicado para a base completa de dados $(n=331)$. Nessa etapa, foi comprovada novamente a unidimensionalidade dos fatores que apresentaram índices de consistência interna acima de 0,70, conforme é recomendado por Pedhazur e Schmelkin (1991). Esses índices de consistência interna estão acima do recomendado por Hair, Anderson, Tatham e Black (1995), Kolenikov, Steinley e Thombs (2010) e Pedhazur e Schmelkin (1991). O próximo passo no tratamento dos dados consistiu na aplicação da Análise Fatorial Confirmatória, via Modelagem de Equações Estruturais.

\section{Análise fatorial confirmatória}

A Análise Fatorial Confirmatória foi realizada com o emprego do método de estimação da Máxima Verossimilhança (ML) (Kolenikov et al., 2010). O modelo da AFC apresentou ótimos índices de ajustamento. Foram consideradas as seguintes medidas: (a) ajuste absoluto (RMR, GFI, RMSEA); (b) ajuste incremental (AGFI, NFI e TLI); (c) ajuste parcimonioso (RFI, IFI e CFI) e; (d) ajuste geral (razão entre Qui Quadrado e Graus de Liberdade).

Considerando as medidas de ajuste absoluto, o RMR (raiz do resíduo quadrático médio) do modelo é de 0,033 . Esse valor, próximo de 0 , indica presença de fortes correlações na matriz dos 
dados. O GFI (índice de qualidade de ajuste) de 0,969 está acima da referência mínima. O RMSEA (raiz do erro quadrático médio de aproximação) é de 0,010 , portanto, abaixo do valor máximo de 0,08 , como é recomendado por Hair et al. (1995) e Kolenikov et al. (2010). O p-value (valor de significância) $=0,402$ atende ao valor esperado $>0,05$ (acima de 0,05 ), que indica não haver diferença entre a matriz prevista e a matriz real dos dados.

As medidas adicionais de ajustamento utilizadas na Análise Fatorial Confirmatória foram o ajuste incremental, parcimonioso e geral. Medidas de ajuste incremental apresentam indicadores comparados a um modelo nulo (modelo de SEM com apenas um fator e sem erro de mensuração) (Kolenikov et al., 2010). A medida AGFI (índice ajustado de qualidade de ajuste) é de 0,953 e o RFI = 0,967 . Os indicadores NFI (índice de ajuste normado) $=0,975$, TLI (índice de ajuste não normado) $=$ 0,999, IFI (índice de ajuste incremental) $=0,999$ e CFI (índice de ajuste comparativo) $=0,999$ também atestam a alta qualidade do modelo. Os valores de $\chi^{2}$ (Qui Quadrado) $=82,488$ e GL (graus de liberdade $)=80$ são utilizados no cálculo do Qui Quadrado Normado. Essa medida $\chi^{2} / \mathrm{GL}=1,031$ está dentro dos limites recomendados de 1 a 3 (Hair et al., 1995).

\section{Validade e confiabilidade das medidas}

Com a aplicação da Análise Fatorial Confirmatória (AFC), foi possível examinar a validade convergente e discriminante das medidas. Essas duas formas de validade de constructo são tidas como as principais por Pedhazur e Schmelkin (1991) e Schumacker e Lomax (2004). A validade convergente foi assegurada pela significância estatística da carga padronizada dos indicadores em seus respectivos fatores. Em todos os casos, o carregamento dos indicadores mostrou-se abaixo de 0,05, ou seja, os indicadores de cada fator convergem na mensuração da mesma variável latente.

Para verificação da validade discriminante, foi adotado o procedimento sugerido por Fornell e Larcker (1981), em que a correlação entre cada um dos constructos é elevada ao quadrado e comparada com a Variância Média Extraída (AVE), que sempre deve ser superior à correlação dos constructos. O AVE de todas as medidas revelou-se acima da correlação dos constructos elevada ao quadrado, o que comprova a validade discriminante dos fatores. A Confiabilidade Composta foi considerada acima do valor mínimo de 0,70 em todos os constructos, como recomenda Hair et al. (1995) e Pedhazur e Schmelkin (1991). Os carregamentos dos indicadores e medidas de confiabilidade constam na Tabela 1.

Tabela 1

Resultados da Análise Fatorial Confirmatória

\begin{tabular}{|c|c|c|c|c|}
\hline Indicador & Carregamento & Confiabilidade & AVE & $\begin{array}{c}\text { Alfa de } \\
\text { Cronbach }\end{array}$ \\
\hline Contracrescimento Percebido 1 & $0,671 \ddagger$ & 0,82 & 0,61 & 0,82 \\
\hline Contracrescimento Percebido 2 & 0,795 € & & & \\
\hline Contracrescimento Percebido 3 & $0,862 *$ & & & \\
\hline Incongruência Percebida 1 & $0,627 \mathrm{t}$ & 0,78 & 0,55 & 0,78 \\
\hline Incongruência Percebida 2 & $0,840 €$ & & & \\
\hline Incongruência Percebida 3 & $0,749 *$ & & & \\
\hline Satisfação 1 & $0,856^{*}$ & 0,89 & 0,75 & 0,90 \\
\hline Satisfação 2 & $0,880 €$ & & & \\
\hline Satisfação 3 & $0,857 \mathrm{z}$ & & & \\
\hline
\end{tabular}


Tabela 1 (continuação)

\begin{tabular}{|c|c|c|c|c|}
\hline Indicador & Carregamento & Confiabilidade & AVE & $\begin{array}{c}\text { Alfa de } \\
\text { Cronbach }\end{array}$ \\
\hline Cooperação 1 & $0,771 *$ & 0,88 & 0,71 & 0,87 \\
\hline Cooperação 2 & 0,902 表 & & & \\
\hline Cooperação 3 & 0,853 毛 & & & \\
\hline Intenção de saída 1 & $0,885^{*}$ & 0,94 & 0,85 & 0,94 \\
\hline Intenção de saída 2 & 0,958 毛 & & & \\
\hline Intenção de saída 3 & 0,927 € & & & \\
\hline
\end{tabular}

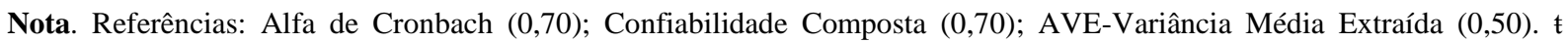
Carregamentos significativos a p-value $<0,01$. Fonte: Elaborada pelos autores.

*Significância não calculada em função de o parâmetro estar afixado em 1 .

As correlações entre os constructos contracrescimento, incongruência percebida, satisfação com o trabalho, intenção de saída e cooperação do indivíduo no trabalho são detalhadas na Tabela 2. Apenas a correlação entre satisfação e incongruência percebida não foi significativa a um $p$-value < 0,001 .

Tabela 2

Correlações entre Variáveis Latentes

\begin{tabular}{lcccc}
\hline & Incongruência & Satisfação & Cooperação & Intenção de Saída \\
\hline Contra crescimento & $0,299^{*}$ & $-0,492^{*}$ & $-0,505^{*}$ & $0,495^{*}$ \\
Incongruência & & $-0,100$ & $-0,012^{*}$ & $0,217^{*}$ \\
Satisfação & & $0,524^{*}$ & $-0,700^{*}$ \\
Cooperação & & & $-0,464^{*}$ \\
\hline
\end{tabular}

Nota. Fonte: elaborada pelos autores.

$*$ p-value $<0,001$

Dadas as demais correlações encontradas entre os constructos investigados, deu-se prosseguimento à aplicação da técnica de Modelagem de Equações Estruturais para avaliação da qualidade do modelo estrutural completo e dos resultados de teste das hipóteses desenvolvidas no referencial teórico-empírico do presente estudo.

\section{Modelo estrutural}

De acordo com Kolenikov et al. (2010), a técnica de modelagem de equações estruturais é uma técnica multivariada bem-estabelecida para problemas que envolvem análise de múltiplas equações ao mesmo tempo. Para avaliar o modelo estrutural proposto inicialmente, foi testado também um modelo rival e, então, as medidas de ajuste de ambos foram comparadas. Como propõem Hair et al. (1995) e Pedhazur e Schmelkin (1991), a abordagem final para avaliação de um modelo estrutural é a lógica de comparação de modelos concorrentes. O modelo alternativo ou rival considera as seguintes relações: a satisfação com o trabalho influencia diretamente incongruência percebida, contracrescimento, intenção de saída e cooperação. E a incongruência e contracrescimento, como no modelo proposto inicialmente, continuam a influenciar intenção de saída e cooperação. Como se observa, a mudança principal centrou no estabelecimento da satisfação como antecedente da incongruência percebida e do contracrescimento, mantendo as demais relações como no modelo proposto inicialmente. 
A julgar pelas medidas de ajuste do modelo proposto e do modelo rival na Tabela 3, é possível advogar em favor do modelo inicial. Apesar de também serem considerados bons os índices do modelo rival, suas medidas são inferiores ao modelo inicial.

Tabela 3

Medidas de Ajuste do Modelo Inicial e Modelo Rival

\begin{tabular}{lccc}
\hline Medida de Ajuste & Modelo Proposto & Modelo Rival & Referência \\
\hline P-value & 0,402 & 0,072 & $>0,050$ \\
Goodness of Fit Index (GFI) & 0,969 & 0,963 & $\geq 0,900$ \\
Adjusted Goodness of Fit Index (AGFI) & 0,953 & 0,945 & $\geq 0,900$ \\
Normed Fit Index (NFI) & 0,975 & 0,969 & $\geq 0,900$ \\
Tucker-Lewis Coefficient (TLI) & 0,999 & 0,992 & $\geq 0,900$ \\
Relative Fit Index (RFI) & 0,967 & 0,960 & $\geq 0,900$ \\
Incremental Fit Index (IFI) & 0,999 & 0,994 & $\geq 0,900$ \\
Comparative Fit Index (CFI) & 0,999 & 0,994 & $\geq 0,900$ \\
Root Mean Square Residual (RMR) & 0,033 & 0,058 & $\leq 0,080$ \\
Root Mean Square Error of Approximation (RMSEA) & 0,010 & 0,027 & $\leq 0,080$ \\
Chi-Square/Degree of Freedom $\left(\chi^{2}\right) /(d f)$ & 1,031 & 1,239 & 1,0 a 3,0 \\
\hline
\end{tabular}

Nota. Fonte: Elaborada pelos autores.

Destacada a avaliação positiva do modelo estrutural, foi examinado cada um de seus parâmetros a partir dos coeficientes padronizados das relações que deram suporte ao teste das hipóteses do levantamento. Os coeficientes padronizados $(\beta)$ e seus níveis de significância são detalhados na Tabela 4.

Tabela 4

\section{Parâmetros do Modelo Estrutural}

\begin{tabular}{llccc}
\hline Parâmetro $(\gamma)$ & $\boldsymbol{B}$ & $\boldsymbol{t}$-value & $\boldsymbol{p}$-value $(\leq)$ \\
\hline H1a) & Incongruência $\rightarrow$ Satisfação & 0,026 & 0,425 & 0,670 \\
H1b) & Contracrescimento $\rightarrow$ Satisfação & $-0,497$ & $-7,778$ & 0,001 \\
H2a) & Incongruência $\rightarrow$ Intenção de Saída & 0,116 & 2,386 & 0,017 \\
H2b) & Contracrescimento $\rightarrow$ Intenção de Saída & 0,171 & 2,983 & 0,003 \\
H3) & Satisfação $\rightarrow$ Intenção de Saída & $-0,615$ & $-10,414$ & 0,001 \\
H4a) & Incongruência $\rightarrow$ Cooperação & 0,126 & 2,227 & 0,026 \\
H4b) & Contracrescimento $\rightarrow$ Cooperação & $-0,332$ & $-4,818$ & 0,001 \\
H5) & Satisfação $\rightarrow$ Cooperação & 0,275 & 3,405 & 0,001 \\
H6) & Intenção de Saída $\rightarrow$ Cooperação & $-0,125$ & $-1,633$ & 0,102 \\
\hline
\end{tabular}

Nota. Fonte: Elaborada pelos autores.

A primeira hipótese $\left(\mathrm{H}_{1 \mathrm{a}}\right)$, que versa sobre a influência negativa da incongruência percebida sobre a satisfação com o trabalho, mostrou que não existe associação estatisticamente significativa entre esses elementos $(\beta=0,026, p$-value $=0,670)$. Tal resultado apontou que essa dimensão da sobrequalificação pode variar positiva ou negativamente e não causar variação significativa sobre a 
satisfação. A hipótese $\left(\mathrm{H}_{1 \mathrm{~b}}\right)$ foi confirmada. De acordo com os resultados $(\beta=-0,497, p$-value $=$ $0,001)$, que associam negativamente contracrescimento e satisfação, verifica-se que a falta de condições organizacionais que estimulem o desafio no trabalho faz com que o indivíduo sinta-se mais insatisfeito. A relação de influência da incongruência sobre a intenção de saída $\left(\mathrm{H}_{2 \mathrm{a}}\right)(\beta=0,116, p$ value $=0,017)$ e do contracrescimento sobre a intenção de saída $\left(\mathrm{H}_{2 \mathrm{~b}}\right)(\beta=0,171, p$-value $=0,003)$ foram corroboradas. Respectivamente, tais confirmações apontam que a intenção de saída é influenciada tanto pela falta de oportunidades de aprendizagem e desafio (contracrescimento) quanto pela distância entre o que o indivíduo faz no trabalho e aquilo que ele poderia fazer em função de suas qualificações (incongruência). Da mesma maneira, a hipótese $\left(\mathrm{H}_{3}\right)$ confirmou a influência da satisfação sobre a intenção de saída com um $\beta$ negativo $(\beta=-0,615, p$-value $=0,001)$, conforme foi previsto. Com a relação mais forte entre as variáveis latentes do modelo estrutural, foi confirmada que a intenção de saída é influenciada principalmente pela satisfação com o trabalho. Em linhas gerais, a intenção de saída e, por consequência, a rotatividade de funcionários estão ligadas ao atendimento das expectativas do indivíduo enquanto realiza seu trabalho.

A hipótese $\left(\mathrm{H}_{4 a}\right)$ que trata da relação entre incongruência e cooperação $(\beta=0,126, p$-value $=$ $0,026)$ assinalou que esses dois constructos estão associados em termos estatísticos, mas com um beta $(\beta)$ positivo ao invés de negativo, como era teoricamente esperado. Esse resultado pode ser puramente espúrio ou indicar uma relação mais complexa entre esses elementos, na qual a incongruência (distância entre o grau de exigência do trabalho e as qualificações do indivíduo) colabora para que com menos esforços o indivíduo faça mais pela organização. Por sua vez, a hipótese que relaciona contracrescimento e cooperação $\left(\mathrm{H}_{4 \mathrm{~b}}\right)$ foi confirmada $(\beta=-0,332$, p-value $=0,001)$. Como estabelecido no desenvolvimento das hipóteses, a falta de desafios e oportunidades de desenvolvimento faz com que o grau de cooperação seja reduzido. A hipótese $\left(\mathrm{H}_{5}\right)$ confirma a influência da satisfação sobre o comportamento cooperativo $(\beta=0,275, p$-value $=0,001)$; e a hipótese $\left(\mathrm{H}_{6}\right)$ não dá suporte à relação causal negativa entre intenção de saída e cooperação $(\beta=-0,125, p$-value $=0,102)$. As últimas hipóteses $\left(\mathrm{H}_{5}\right.$ e $\left.\mathrm{H}_{6}\right)$, respectivamente, apontam que o grau de cooperação depende da satisfação das necessidades do indivíduo em relação ao trabalho, mas revelam que a intenção de saída não altera o comportamento cooperativo. Tais achados são ainda mais interessantes quando se nota que as duas dimensões da sobrequalificação (incongruência e contracrescimento) apresentam influência estatisticamente significativa sobre a cooperação, apontando maior importância da percepção de qualificação em comparação a outros fatores relacionados ao comportamento no trabalho. Os coeficientes padronizados $(\beta)$ dessas relações causais são apresentados no modelo estrutural na Figura 2.

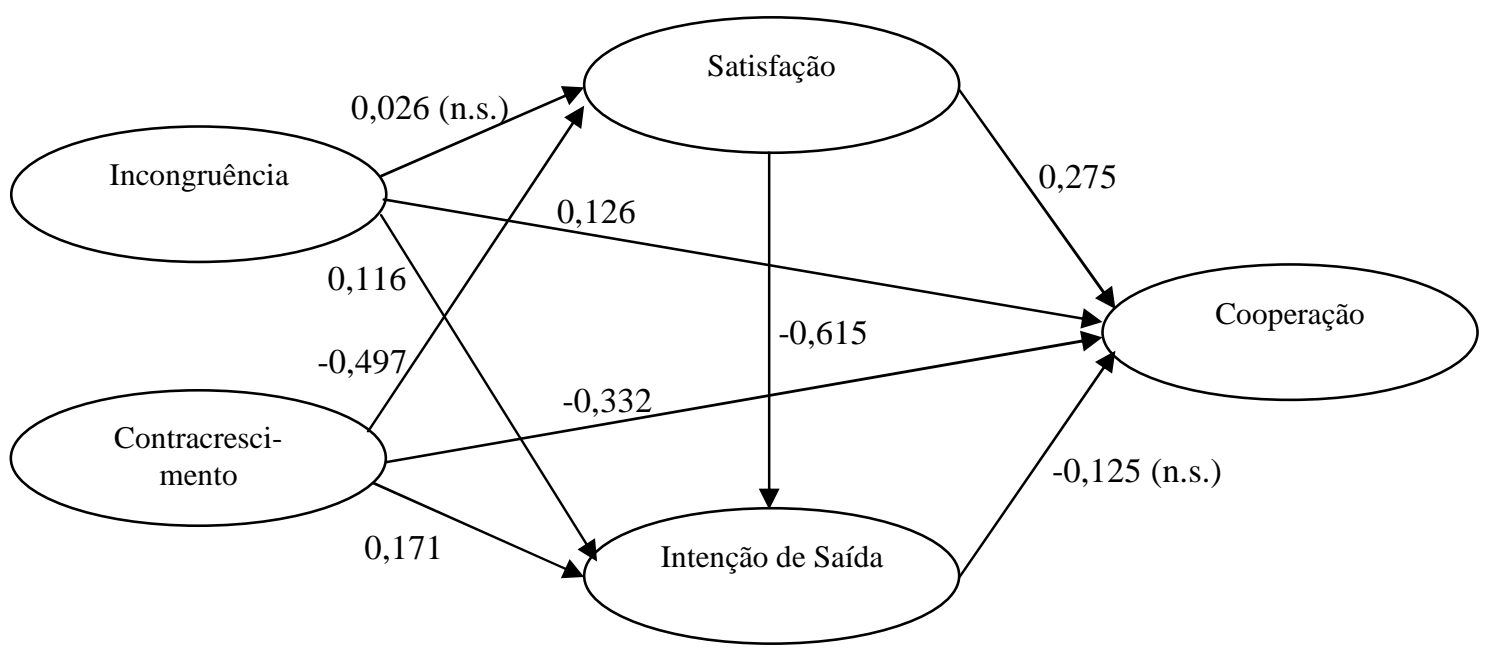

Figura 2. Modelo Estrutural Testado.

n.s: Relação não significativa ( $p$-value $<0,05)$. Fonte: Elaborada pelos autores. 


\section{Discussão dos Resultados}

As justificativas teóricas construídas para desenvolvimento da primeira hipótese $\left(\mathrm{H}_{1 \mathrm{a}}\right)$ sustentaram a ideia de que, ao perceber que suas habilidades pessoais estão acima das necessidades da função exercida, o indivíduo apresenta tendência de redução da satisfação com o trabalho. Contudo os resultados não deram suporte, em termos estatísticos, para essa hipótese. Apesar de pesquisadores como McKee-Ryan e Harvey (2011) e Scurry e Blenkinsopp (2011) destacarem que a sobrequalificação fatalmente produz consequentes negativos sobre atitude e comportamento do indivíduo no trabalho, isso não foi confirmado no presente estudo. Como apontado, não foi verificada associação estatisticamente significativa entre incongruência percebida e satisfação no trabalho. Tal achado pode sugerir que, apesar de o indivíduo ter consciência de que suas qualificações estão acima do necessário, ele compreende que isso não justifica um sentimento de insatisfação. A culpa por ele ser qualificado demais não seria da organização, aos olhos do próprio funcionário (G. J. Johnson \& Johnson, 2000). É interessante observar, a respeito, Fine (2007) e Fine e Nevo (2008), os quais argumentam que a maior oferta e consequente qualificação do ensino superior gera maior sobrequalificação, mas não necessariamente esse movimento de socialização do ensino superior produzirá indivíduos mais insatisfeitos com o trabalho.

Por consequência, a explicação para a variação da satisfação com o trabalho desloca-se para outros fatores, no caso do presente estudo, mais especificamente para a dimensão contracrescimento do constructo sobrequalificação, caso da hipótese $\left(\mathrm{H}_{1 \mathrm{~b}}\right)$, que estabelece uma relação negativa entre a falta de oportunidade dada pela organização para uso e desenvolvimento de novas habilidades e a satisfação. De acordo com G. J. Johnson e Johnson (1999), condições organizacionais de estrutura, rotinas e aspectos culturais operam como base para a percepção de sobrequalificação do tipo Contracrescimento. Assim, como essa hipótese $\left(\mathrm{H}_{1 \mathrm{~b}}\right)$ foi corroborada, é possível afirmar que a influência da percepção de sobrequalificação sobre a satisfação com o trabalho reside prioritariamente na capacidade de a organização estimular o desenvolvimento do funcionário (G. J. Johnson \& Johnson, 2000). W. R. Johnson et al. (2002) chamam de oportunidades promocionais todas as alternativas que são providas pela organização para desenvolvimento de habilidades no trabalho. Ainda de acordo com esses autores, a falta de condições desafiadoras de trabalho leva o indivíduo ao movimento de deterioração do valor do trabalho e de baixos índices de investimento na carreira em determinada organização.

Em outras palavras, se o indivíduo tem qualificações acima de sua função, isso não afeta sua satisfação, mas, se a organização impede que ele desenvolva novas habilidades, há um efeito direto sobre a sua avaliação global do trabalho. O ponto de partida da avaliação da utilização ou não das habilidades (G. J. Johnson \& Johnson, 1999, 2000) muda o efeito da sobrequalificação sobre a satisfação global (Ferrat, 1981). Apesar de G. J. Johnson e Johnson (1999, 2000) destacarem o Contracrescimento Percebido (No-Growth) e a Incongruência (Mismatch) como duas dimensões reflexivas e, sobretudo, correlacionadas, do constructo sobrequalificação, os resultados do presente estudo mostram que essa associação não é tão acentuada (Coeficiente de Correlação $=0,299$ ). Tal achado abre espaço para que tais dimensões apresentem correlação diferenciada quando considerado um mesmo constructo consequente ou antecedente da sobrequalificação.

Em relação à influência da incongruência percebida e do contracrescimento (W. R. Johnson et al., 2002) sobre a intenção de saída, o resultado dos testes das hipóteses $\left(\mathrm{H}_{2 \mathrm{a}}\right)$ e $\left(\mathrm{H}_{2 \mathrm{~b}}\right)$ confirmaram que as duas dimensões da sobrequalificação conduzem à intenção de se desligar da organização. Desse modo, a contratação de funcionários com qualificações acima do exigido pela função e ainda a falta de oportunidades de desenvolvimento de novas habilidades se somam para que ocorra um aumento na intenção de sair da organização. Tais achados corroboram os argumentos de W. R. Johnson et al. (2002) de que características organizacionais e desencontros entre habilidades e tarefas levam a comportamentos contraproducentes aos investimentos organizacionais. Nesse sentido, o resultado da hipótese $\left(\mathrm{H}_{1 \mathrm{a}}\right)$, sem associação estatisticamente significativa, que versa sobre a relação entre incongruência percebida e satisfação, é ainda mais interessante, pois o funcionário não tem redução na 
sua satisfação em função da dimensão incongruência percebida, mas, de qualquer maneira, ele procurará provavelmente outra organização para trabalhar. Destaca-se ainda que o fator contracrescimento apresentou um coeficiente padronizado levemente superior comparado ao fator incongruência.

No que concerne à relação causal satisfação $\rightarrow$ intenção de saída, o teste da hipótese $\left(\mathrm{H}_{3}\right)$ apresenta o maior coeficiente padronizado $(\beta=-0,615)$, portanto o efeito direto mais significativo do modelo estrutural está entre essas variáveis, o que só vem a confirmar uma asserção já bemestabelecida na literatura (Cotton \& Tuttle, 1986; Mobley, 1982), mas que não havia sido testada em meio às demais hipóteses aqui contempladas. Entretanto, apesar de já documentada, há de se destacar que tal associação é potencializada pela dimensão Contracrescimento no modelo estrutural testado. Isso ocorre na medida em que o Contracrescimento influencia pouco a intenção de saída, mas mais acentuadamente a satisfação; e esta influencia mais significativamente a intenção de saída.

Sobre a influência da incongruência percebida no comportamento cooperativo $\left(\mathrm{H}_{4 \mathrm{a}}\right)$, é importante destacar que, curiosamente essa hipótese apresentou coeficiente padronizado $(\beta)$ no sentido contrário ao esperado (positivo), com nível de significância estatística ( $p$-value) $<0,05$. O resultado do modelo estrutural indica que quanto maior a percepção de que o indivíduo possui habilidades além do necessário para execução de sua função maior será sua cooperação. Uma provável razão para essa aparentemente contraditória relação, talvez, resida no fato de que se esses indivíduos são, como se percebem, efetivamente qualificados além do necessário, a cooperação varie positivamente em função da sua capacidade, e não da volição do trabalhador em cooperar ou não. Vale chamar atenção a essa contribuição do presente trabalho à medida que a sobrequalificação aparece exclusivamente na literatura consultada com conotação negativa. A influência positiva da sobrequalificação sobre o comportamento não foi considerada nos trabalhos de G. J. Johnson e Johnson (1999, 2000, 2002). Entretanto não se deve desconsiderar também a possibilidade de que simplesmente a relação entre esses elementos seja espúria. De qualquer modo, essa seria uma oportunidade para o desenvolvimento de futuros levantamentos, talvez, até combinados com pesquisas de cunho qualitativo ou ainda experimentos para a busca de novas explanações.

Já o resultado do efeito causal contracrescimento $\rightarrow$ cooperação $\left(\mathrm{H}_{4 \mathrm{~b}}\right)$, confirma a ideia de que o comportamento cooperativo no trabalho varia negativamente em função da incapacidade da organização em explorar suficientemente as qualificações do indivíduo no trabalho, bem como em função de dar espaço para o desenvolvimento dessas e novas habilidades. Tal achado fortalece a lógica exposta por G. J. Johnson e Johnson $(2000$, 2002) de que fatores organizacionais são aspectos centrais no aumento ou redução da sobrequalificação. Na mesma linha de raciocínio, as conclusões do trabalho de Erdogan e Bauer (2009) esclarecem como táticas de delegação de poder influenciam a percepção de desafio no trabalho e, por conseguinte, a sobrequalificação.

Em meio às demais relações do modelo estrutural proposto, a influência da satisfação do indivíduo no trabalho sobre seu comportamento cooperativo $\left(\mathrm{H}_{5}\right)$ evidencia a noção de Barnard (1979), ou seja, de que o atendimento das necessidades dos membros organizacionais opera como antecedente direto da cooperação. Barnard (1979, p. 80) esclarece que: "Se um indivíduo acha que seus motivos estão sendo satisfeitos pelo que ele faz, ele continua o seu esforço cooperativo; em caso contrário, ele não o faz".

$\mathrm{O}$ resultado da última hipótese $\left(\mathrm{H}_{6}\right)$ do modelo estrutural, não corrobora a ideia de que a intenção de saída da organização influencia negativamente o comportamento cooperativo do indivíduo no trabalho. Tal achado revela ausência de alteração da ação cooperativa em função do objetivo de deixar a organização. Assim, é possível afirmar que, independentemente do grau de intenção de se desligar da organização, a cooperação ocorre na mesma intensidade, sendo influenciada, então, por outros fatores. De acordo com Maciel e Camargo (2011), o desempenho, em termos de comportamento cooperativo, pode não sofrer variação em função de algumas das atitudes do indivíduo por causa das normas de reciprocidade. Essas normas de reciprocidade são os comportamentos que o funcionário acha que terceiros ou a empresa esperam dele. Esse padrão de expectativas de comportamento seria mais forte do que a influência da intenção de saída sobre a cooperação. 
Em resumo, considerando as dimensões da sobrequalificação, a insatisfação do funcionário, a intenção de saída e a baixa cooperação, essas são resultados da comparação que o indivíduo faz entre suas habilidades e as oportunidades de desenvolvimento providas pela organização. Assim, as dimensões da sobrequalificação apresentam-se como constructos importantes para ampliação das investigações acerca das interações entre elementos atitudinais e comportamentais.

\section{Considerações Finais}

Antes de destacar os principais achados da pesquisa e suas implicações, é importante ressaltar as limitações que marcaram o desenvolvimento do trabalho. Algumas dificuldades têm origem no baixo número de artigos pertinentes ao conceito sobrequalificação, o que possivelmente ocorre em função da notoriedade do constructo competência em âmbito individual e organizacional, como anteriormente mencionado. Outros fatores que exigem cautela na leitura dos resultados repousam no procedimento amostral do estudo e nas escalas que foram propostas aqui. Utilizou-se uma amostragem não probabilística por conveniência, o que restringe qualquer possibilidade de generalização estatística. Quanto às escalas, ocorreu a tradução dos indicadores para mensuração das dimensões da sobrequalificação e geração de novos itens para avaliação dos demais constructos. Por sua vez, podese destacar a qualidade do modelo proposto em termos de medidas de ajustamento, sobretudo em comparação a um modelo rival. Em adição, destaca-se que entre os questionários excluídos após a coleta de dados a maior parte não apresentou respostas para as características desses indivíduos. Isso impossibilitou a realização de um teste para verificar diferenças significativas entre aqueles que terminaram de responder o questionário e os que optaram por abortar o preenchimento. Tal limitação impõe considerar a possibilidade de viés de seleção. Portanto, mais uma vez é preciso alertar que não é possível realizar qualquer generalização estatística. Contudo vale destacar que essa preocupação com a generalização estatística e com o viés de seleção são resquícios positivistas do produzir ciência, pois na medida em que não se busca realizar previsões ou dar orientações para a empresa, por não se tratar de um trabalho de consultoria, é priorizada aqui a generalização analítica - o que se pode "de fato concluir" para a teoria que orientou o presente trabalho e não para a população do estudo.

Considera-se, a partir dos achados, que existe maior influência da dimensão contracrescimento (No-Growth) (W. R. Johnson et al., 2002) da sobrequalificação, sobre constructos atitudinais e comportamentais, em comparação aos efeitos da dimensão incongruência sobre as mesmas variáveis e, portanto, a maior responsabilidade pela percepção do grau de subutilização das habilidades do indivíduo é das próprias organizações de trabalho. Assim, salienta-se que mudanças em variáveis controladas pelas organizações podem atenuar as avaliações e sentimentos de privação no que concerne à utilização do pleno potencial para o trabalho.

Tal consideração foi delimitada a partir da constatação de que o atendimento das necessidades (i.e., satisfação) do indivíduo é substancialmente influenciado pela dimensão contracrescimento (NoGrowth). Da mesma maneira, o contracrescimento apresenta mais forte influência sobre a intenção de saída em comparação à incongruência percebida (Mismatch). E o mesmo se verifica quando são comparados os efeitos das duas dimensões da sobrequalificação (No-Growth/Mismatch) sobre o comportamento cooperativo no trabalho.

Ao destacar, no presente artigo, a responsabilidade da própria organização acerca da percepção de sobrequalificação (overqualification) dos indivíduos no trabalho, não se pretende desconsiderar fatores ambientais (e.g., recessão econômica) como seus antecedentes. Todavia cabe apontar que, entre características individuais e organizacionais, as características de natureza organizacional apresentamse como fatores mais importantes de influência ou moderação (Erdogan \& Bauer, 2009) dos efeitos da sobrequalificação sobre variáveis atitudinais e comportamentais. Isso significa a possibilidade e também a necessidade de uma agenda de pesquisa para investigação da sobrequalificação que contemple as relações entre esse constructo e variáveis como estrutura organizacional, tecnologia (processos organizacionais), empowerment e aprendizagem. 
Em adição, futuros estudos nesta linha podem ajudar também a esclarecer a relação positiva entre incongruência e a cooperação do indivíduo no trabalho. Especulou-se aqui a possibilidade de essa variação ocorrer no sentido positivo em função da própria qualificação, pois quanto mais qualificado o indivíduo, mesmo com a percepção de sobrequalificação, estaria apresentando comportamento cooperativo mais intenso. Entretanto, à medida que a incongruência entre as habilidades e as exigências funcionais é superada em função da capacidade superior do funcionário na organização, os efeitos dessa relação tendem a ser reduzidos por conta da segunda dimensão da sobrequalificação no trabalho - contracrescimento (No-Growth).

\section{Referências}

Anda, R. M., \& Sobczak, M. (2011). Underemployment among Mexican-origin women. Social Science Journal, 48(4), 621-629. doi: 10.1016/j.soscij.2011.03.005

Axelrod, R., \& Dion, D. (1988). The further evolution of cooperation. Science, 242(4884), 1385-1390. doi: $10.1126 /$ science.242.4884.1385

Babbie, E. R. (1998). The practice of social research. California: Wadsworth Publishing.

Barnard. C. I. (1979). As funções do executivo. São Paulo: Atlas.

Bowen, D. E. (1982). Some unintended consequences of intention to quit. Academy of Management Review, 7(2), 205-211. doi: 10.5465/AMR.1982.4285563

Brynin, M., \& Longhi, S. (2009). Overqualification: major or minor mismatch? Economics of Education Review, 18(1), 114-121. doi: 10.1080/00036846.2011.554380

Budría, S. (2011). Are educational mismatches responsible for the 'inequality increasing effect' of education? Social Indicators Research, 102(3), 409-437. doi: 10.1007/s11205-010-9675-7

Castro, P. M. R., \& Borges-Andrade, J. E. (2004). Identificação das necessidades de capacitação profissional: o caso dos assistentes administrativos da Universidade de Brasília. Revista de Administração da Universidade de São Paulo, 39(1), 96-108.

Chatman, J. A., \& Barsade, S. G. (1995). Personality, organizational culture and cooperation: evidence from a business simulation. Administrative Science Quarterly, 40(3), 423-443. doi: 10.1037/0021-9010.93.3.702

Chen, X. P., Xie, X., \& Chang, S. (2011). Cooperative and competitive orientation among Chinese people: scale development and validation. Management and Organization Review, 7(2), 353379. doi: 10.1111/j.1740-8784.2011.00215.x

Cotton, J. L., \& Tuttle, J. M. (1986). Employee turnover: a meta-analysis and review with implications for research. Academy of Management Review, 11(1), 55-70. doi: 10.5465/AMR.1986.4282625

Dukerich, J. M., Golden, B. R., \& Shortell, S. M. (2002). Beauty is in the eye of the beholder: the impact of organizational identification, identity, and image on the cooperative behaviors of physicians. Administrative Science Quarterly, 47(3), 507-533. doi: 10.2307/3094849

Erdogan, B., \& Bauer, T. N. (2009). Perceived overqualification and its outcomes: the moderating role of empowerment. Journal of Applied Psychology, 94(2), 557-565. doi: 10.1037/a0013528

Ferrat, T. W. (1981). Overall job satisfaction: is it a linear function of facet satisfaction? Human Relations, 34(6), 463-473. doi: 10.1177/001872678103400603 
Fine, S. (2007). Overqualification and selection in leadership training. Journal of Leadership \& Organizational Studies, 14(1) 61-68. doi: 10.1177/1071791907304291

Fine, S., \& Nevo, B. (2008). Too smart for their own good? A study of perceived cognitive overqualification in the workforce. The International Journal of Human Resource Management, 19(2), 346-355. doi: 10.1080/09585190701799937

Frei, C., \& Sousa-Poza, A. (2012). Overqualification: permanent or transitory? Applied Economics, 44(14), 1837-1847. doi: 10.1080/00036846.2011.554380

Fornell, C., \& Larcker, D. F. (1981). Evaluating structural equation models with unobserved variables and measurement error. Journal of Marketing Research, 18(1), 39-50. doi: 10.2307/3150980

Green, F., \& Zhu, Y. (2010). Overqualification, job dissatisfaction, and increasing dispersion in the returns to graduate education. Oxford Economic Papers, 62(4), 740-763. doi: 10.1093/oep/gpq002

Hair, J. F., Jr., Anderson, R. E., Tatham, R. L., \& Black, W. C. (1995). Multivariate data analysis. New Jersey: Prentice-Hall, Inc.

Johnson, W. R., Morrow, P. C., \& Johnson, G. J. (2002). An evaluation of a perceived overqualification scale across work settings. The Journal of Psychology, 136(4), 425-441. doi: $10.1080 / 00223980209604169$

Johnson, G. J., \& Johnson, W. R. (1999). Perceived overqualification and health: a longitudinal analysis. The Journal of Social Psychology, 139(1), 14-28. doi: 10.1080/00224549909598358

Johnson, G. J., \& Johnson, W. R. (2000). Perceived overqualification and dimensions of job satisfaction: a longitudinal analysis. The Journal of Psychology, 134(5), 537-555. doi: $10.1080 / 00223980009598235$

Johnson, G. J., \& Johnson, W. R. (2002). Perceived overqualification, positive and negative affectivity and satisfaction with work. Journal of Social Behavior and Personality, 15(2), 167-184.

Jones, G. R., \& George, J. M. (1998). The experience and evolution of trust: implications for cooperation and teamwork. Academy of Management Review, 23(3), 531-546. doi:10.2307/259293

Kerlinger, F. N. (2003). Metodologia da pesquisa em ciências sociais. São Paulo: Editora Pedagógica e Universitária.

Kolenikov, S., Steinley, D., \& Thombs, L. (2010). Statistics in the social sciences. New Jersey: Wiley.

Laschinger, H. K. S., Finegan, J. E., Shamian, J., \& Wilk, P. (2004). A longitudinal analysis of the impact of workplace empowerment on work satisfaction. Journal of Organizational Behavior, 25(4), 527-545. doi: 10.1002/job.256

Livingstone, D. W. (2010). Job requirements and worker`s learning: formal gaps, informal closure, systemic limits. Journal of Education and Work, 23(3), 207-231. doi: $10.1080 / 13639081003785732$

Maciel, C. O., \& Camargo, C. (2011). Comprometimento, satisfação e cooperação no trabalho: evidências da primazia dos aspectos morais e das normas de reciprocidade sobre $\mathrm{o}$ comportamento. Revista de Administração Contemporânea, 15(3), 433-453. Recuperado de http://www.scielo.br/pdf/rac/v15n3/v15n3a05.pdf. doi: 10.1590/S1415-65552011000300005

Mansfield, R. S. (1996). Building competency models: approaches for HR professionals. Human Resource Management, 35(1), 7-18. doi: 10.1002/(SICI)1099-050X(199621)35:1<7::AIDHRM1>3.0.CO;2-2 
Mckee-Ryan, F. M., \& Harvey, J. (2011). "I have a job, but...": a review of underemployment. Journal of Management, 37(4), 962-996. doi: 10.1177/0149206311398134

Mobley, W. H. (1982). Some unanswered questions in turnover and withdrawal research. Academy of Management Review, 7(1), 111-116. doi: 10.5465/AMR.1982.4285493

Noor, K. B. M., \& Dola, K. (2009). Job competencies for malaysian managers in higher education institution. Asian Journal of Management and Humanity Sciences, 4(4), 226-240.

Pedhazur, E., \& Schmelkin, L. (1991). Measurement, design and analysis. Hillsdale: LEA Publisher.

Phua, F. T. T. (2004). The antecedents of co-operative behaviour among project team members: an alternative perspective on an old issue. Construction Management and Economics, 22(10), 1033-1045. doi: 10.1080/01446190310001649092

Rusbult, C. E., \& Farrell, D. (1983). A longitudinal test of the investment model: the impact on job satisfaction, commitment, and turnover of variations in rewards, costs, alternatives and investments. Journal of Applied Psychology, 68(3), 429-438. doi: 10.1037/0021-9010.68.3.429

Sandberg, J. (2000). Understanding human competence at work: an interpretative approach. Academy of Management Journal, 43(1), 9-25. doi: 10.2307/1556383

Schumacker, R. E., \& Lomax, R. G. (2004). A beginner's guide to structural equation modeling. Mahwah: Lawrence Erlbaum Associates Publishers.

Schwepker, C. H., Jr. (2001). Ethical climate's relationship to job satisfaction, organizational commitment, and turnover intention in the salesforce. Journal of Business Research, 54(1), 3952. doi: 10.1016/S0148-2963(00)00125-9

Scurry, T., \& Blenkinsopp, J. (2011). Under-employment among recent graduates: a review of the literature. Personnel Review, 4(5), 643-659. doi: 10.1108/00483481111154487

Spector, P. E. (2005). Introduction: the disposicional approach to job satisfaction. Journal of Organizational Behavior, 26(1), 57-58. doi: 10.1002/job.297

Steel, R. P. (2002). Turnover theory at the empirical interface: problems of fit and function. Academy of Management Review, 27(3), 346-360. doi: 10.5465/AMR.2002.7389900

Sugiyarto, G. (2008). Measuring underemployment: does the cut-off point really matter? Journal of the Asia Pacific Economy, 13(4), 481-517. doi: 10.1080/13547860802364786

Walling, A., \& Clancy, G. (2010). Underemployment in the UK labor market. Economic \& Labour Market Review, 4(2), 16-24. doi: 10.1057/elmr.2010.21

Watt, J., \& Hargis, M. (2010). Boredom proneness: its relationship with subjective underemployment, perceived organizational support, and job performance. Journal of Business \& Psychology, 25(1), 163-174. doi: 10.1007/s10869-009-9138-9

Weststar, J. (2009). Worker control as a facilitator in the match between education and jobs. British Journal of Industrial Relations, 47(4), 723-740. doi: 10.1111/j.1467-8543.2009.00737.x

Whitman, D. S., Rooy, D. L. V., \& Viswesvaran, C. (2010). Satisfaction, citizenship behaviors, and performance in work units: a meta-analysis of collective construct relations. Personnel Psychology, 63(1), 41-81. doi: 10.1111/j.1744-6570.2009.01162.x

Wilkins, R. (2009). The consequences of underemployment for the underemployed. Journal of Industrial Relations, 49(2), 247-275. doi: 10.1177/0022185607074921 


\section{APÊNDICE}

\section{Indicadores do Questionário}

Constructo: Sobrequalificação - Contracrescimento Percebido (Perceived No-Growth) Meu trabalho frequentemente me oferece novos desafios. (indicador invertido). Meu trabalho dá oportunidades para apreender coisas novas (indicador invertido). As tarefas do dia a dia do meu trabalho raramente mudam.

Constructo: Sobrequalificação - Incongruência Percebida (Perceived Mismatch) Minha educação formal me qualifica além do necessário para fazer meu trabalho. Minha experiência profissional está além do que é exigido em meu trabalho atual. Francamente, estou qualificado além do necessário para fazer o meu trabalho.

Constructo: Satisfação

Estou muito satisfeito com a organização na qual trabalho.

Para mim, a organização em que trabalho é muito próxima da "organização ideal". Para mim, a organização em que trabalho é muito melhor do que esperado.

Constructo: Intenção de Saída (Turnover)

Tenho intenção de ir trabalhar em breve numa outra organização.

Frequentemente tenho pensado em mudar de emprego.

Tenho intenção de sair da empresa.

Constructo: Cooperação

Olhando meus colegas de trabalho que mais contribuem com a empresa vejo que estou longe de cooperar com a empresa tanto quanto eles cooperam (indicador invertido).

Estou cooperando com a empresa em $100 \%$ da minha capacidade.

Estou realizando meu trabalho tão bem quanto aquelas pessoas que mais cooperam para alcançar os objetivos da organização. 